\title{
The state of flux
}

\author{
The development of new membrane materials for chemical separations is progressing rapidly, and their \\ commercial success will require a more concerted effort from academia and industry.
}

There are a variety of practical techniques available to achieve the separation of one chemical from another. Obvious examples include crystallization and distillation, which, on a small scale at least, are very simple and rapid processes that can usually be performed with only small amounts of heating required. Where separation techniques such as these become significantly more challenging is at meaningful industrial scales, and satisfying their energy demands can come at an enormous financial and technological expense. The use of membrane materials offers a potentially attractive alternative to achieve these kinds of chemical separations, with what can be substantially reduced resource requirements. Indeed, current industrial-scale separations such as water purification (pictured) and the processing of natural gas already rely heavily on the performance capabilities of membrane materials. In this Focus issue of Nature Materials, we take a detailed look into the current state-of-the-art membrane materials for application in gas- and liquidphase separations and purifications, and consider those particularly promising materials that should be a focus of future investigations. We also discuss important issues of scalability, and how best to transition these new membrane materials from the lab bench to larger-scale operation.

Fundamental considerations for choosing the ideal membrane are the physical and chemical characteristics of the material used, which dictate how well the membrane can selectively remove a specific component in a liquid or gas mixture. On page 289 of this issue, William Koros and Chen Zhang provide a detailed report of the membrane materials and technologies that show the most promise in this regard, with a specific emphasis on molecularly selective membranes ${ }^{1}$. Molecular selectivity is the discrimination between similarly sized molecules in gas and liquid mixtures, and is quite unlike a simpler filtration used to separate dissimilar species. This kind of molecular discrimination is crucial for advanced membrane-based applications in processing mixtures such as nitrogen/oxygen, carbon dioxide/methane, or liquid hydrocarbons. On page 276 Ryan Lively and David Sholl discuss how they believe historical achievements applying membranes

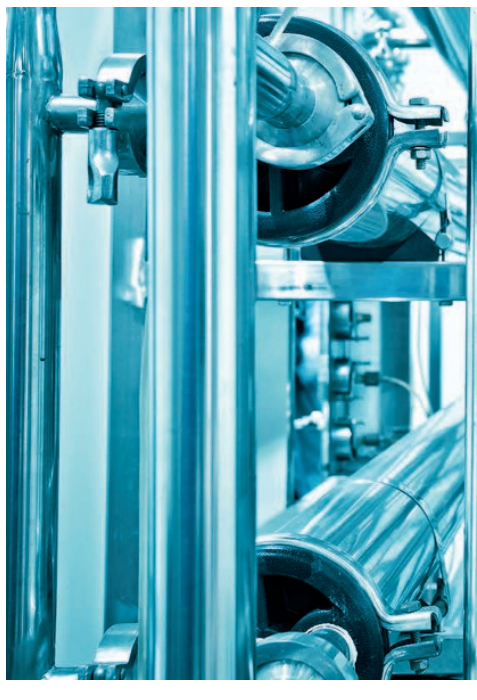

in water purification technologies (for desalination and remediation, for instance) can inspire new and economical membrane systems for the separation of important fluidphase organic molecules ${ }^{2}$.

There is clearly much academic interest in fundamental membrane development, and there is no doubt more still to be learnt through smaller-scale membrane studies in research laboratories. However, relatively few investigations complete the developmental journey to industry and there is a need for more significant efforts to investigate the larger-scale efficiency and operational performance of promising new membrane materials. Such a transition to the market will undoubtedly require close collaboration between academic and industry partners. The new Barrer Centre (www.imperial.ac.uk/barrer-centre) at Imperial College London provides an interesting model of how to foster these kinds of partnerships. Among those in attendance at the centre's launch event in October 2016, the main topic of discussion was how it might achieve - as its main objectives - paradigmchanging membrane and adsorption science and technology, and how this research can be translated into beneficial impacts on industry and society. "Do something useful!" was a popular statement of intent circulating at the event itself, and a clear indication that their research would have practical utility as a prerequisite. On page 280 we interview
Andrew Livingston (Imperial College London and Barrer Centre Director) and Richard Baker (Membrane Technology and Research and Chair of the Barrer Centre Advisory Board) to gain their insights into this kind of collaborative membrane research, on how they hope the Barrer Centre might achieve its aims, and how others should structure their research in order to achieve industrial translation ${ }^{3}$.

What specific materials will form a paradigm-changing membrane is difficult to predict, and practically validating such speculation would take considerable effort, but perhaps the work by Falcaro et al. in this issue (page 342) could serve as one example worthy of further investigation ${ }^{4}$. They show how thin layers of polycrystalline metal-organic frameworks can be grown in such a way that crystal porosity can be aligned over large areas. Beyond this particularly impressive fabrication, it remains to be seen whether these specific materials can offer real performance advantages for chemical separations. Nevertheless, there are clearly gains to be made by those wishing to explore the possible practical capabilities of these and other types of new materials for membrane-based separations.

\footnotetext{
References

1. Koros, W. J. \& Zhang, C. Nat. Mater. 16, 289-297 (2017).

2. Lively, R. P. \& Sholl, D. S. Nat. Mater. 16, 276-279 (2017)

3. Nat. Mater. 16, 280-282 (2017).

4. Falcaro, P. et al. Nat. Mater. 16, 342-348 (2017).
} 